It seems reasonable to combine all these phenomena (which necessarily do not all occur together) into the term 'Solar Storm'. It will be difficult to understand a flare without handling it as a part of such a comprehensive solar event.

The following diagram represents a model of such a Solar Storm and its different components:

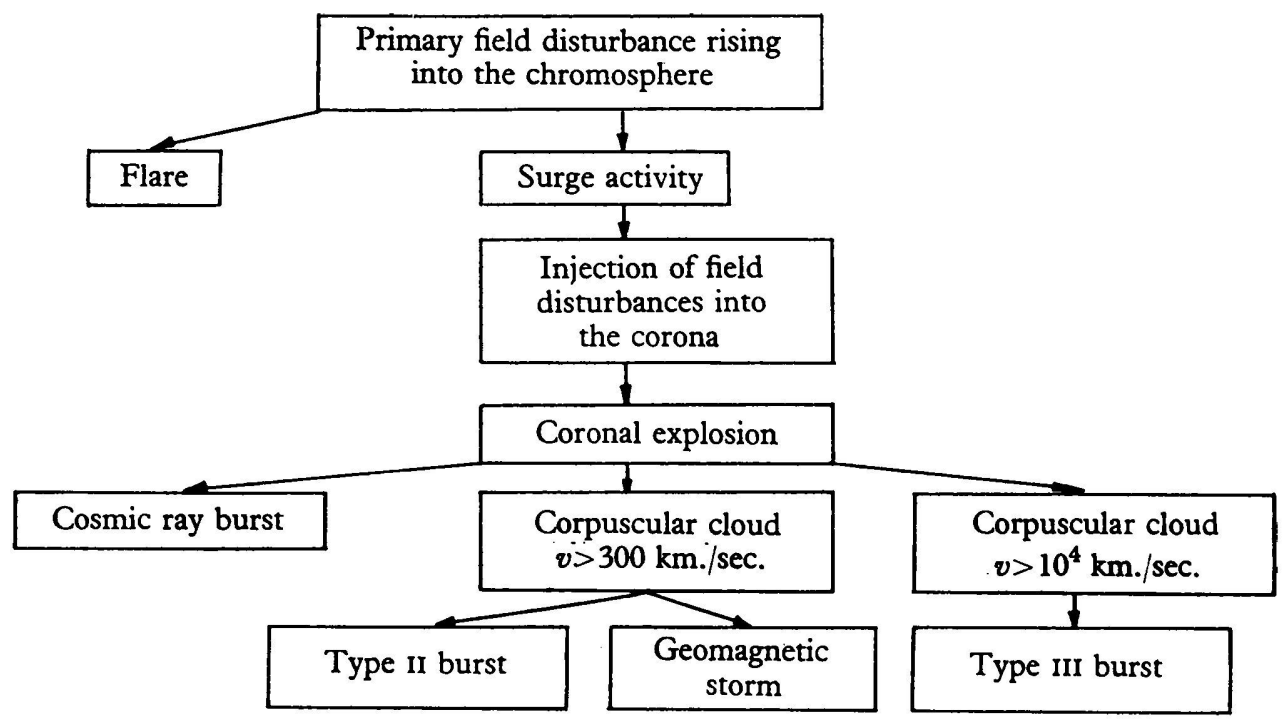

\title{
13. CONTRIBUTION TO THE DISCUSSION
}

By E. R. MUSTEL

I should like to make two remarks on the physics of solar flares. One very interesting problem is the origin of the bright continuous spectrum of a great chromospheric flare.

In a recent paper on this subject I considered two mechanisms. The first one is the recombination, connected with captures of electrons to different levels in the hydrogen atom. In certain cases I found the energy emitted in this process to be sufficient to explain the continuous spectrum sometimes observed. However, this explanation meets the following difficulty: The mechanism leads to a very high intensity of the flares in the Lyman-continuum, in fact, too high to be consistent with the ionospheric observations. This problem requires further consideration.

The second explanation of the continuous spectrum seems to me the most probable one and can be described as follows: We know that the highly luminous matter of chromospheric flares occupies several levels in the solar atmosphere. Extreme cases are the photospheric $\mathrm{H} \alpha$ bombs first described by Ellerman and the flares appearing in coronal space. If we assume that the base of a very great chromospheric flare is situated somewhere in the photosphere, we may expect the temperature in this part of the photophere to increase during the flare. An increase in temperature of only $200^{\circ}$ is quite sufficient for explaining the observed continuous spectrum.

The other of my two remarks concerns the important problem involved in the excitation of atoms in solar flares. I shall consider here only the bright $\mathrm{H}$ and $\mathrm{K}$ lines of $\mathrm{Ca}$ II. In my papers on the physics of chromospheric faculae I have shown that the intensity of the ultra-violet doublet 3706 and 3737 of $\mathrm{Ca}$ II may be considered a sensitive criterion of the mechanism of excitation. If this mechanism is recombination, the $\mathrm{H}$ and $\mathrm{K}$ lines and the ultra-violet doublet will have comparable intensity. But if the mechanism is electronic impact, the intensity of the ultra-violet doublet must be much smaller than 
that of the $\mathrm{H}$ and $\mathrm{K}$ lines. Spectra of chromospheric faculae obtained by me have shown that electronic impact is the principal mechanism here. The observational material which is available for chromospheric flares indicates the same mechanism in these objects, but further observations are needed for conclusive evidence.

\section{CONTRIBUTION TO THE DISCUSSION}

\section{By M. WALDMEIER}

\section{Coronal observations in connexion with Solar Flares}

Limb flares give an opportunity to observe what happens in the solar corona before, during and after the outbreak of a flare. Without exceptions there always appear coronal condensations around the flare material. These bright condensations are best seen in the monochromatic light of the line $5303 \AA$. The line $6374 \AA$ shows less conspicuous features. Especially close is the connexion between the emission of the yellow line $5694 \AA$ and the appearance of the flare. This line brightens up together with the flare, but its intensity is still increasing when the flare fades away. Maximum intensity may be reached about half-an-hour after the beginning of the flare, at a time when $\mathrm{H} \alpha$-emission has completely vanished. At that time generally also the line $5445 \AA$ appears for a short duration. As the production of cosmic radiation starts about half-an-hour after the beginning of the flare, it may be that it is generated in the highly ionized coronal condensation.

A limb flare shows striking similarities with typical sunspot prominences. In many cases it has been observed, that coronal condensations are becoming brighter and denser. By these processes the condensation radiates away its energy and its temperature decreases. After this process has been in progress for about ro-3o min., in the very centre of the condensation a bright point of $\mathrm{H} \alpha$-emission comes into existence. It grows and brightens rapidly, becoming a typical sunspot prominence and after a further quarter-of-an-hour the material is streaming out, downwards into a sunspot. Similar coronal phenomena are observed in connexion with flares. 